Article

\title{
Coefficients Calculation in Pascal Approximation for Passive Filter Design
}

\author{
George B. Kasapoglu ${ }^{1}$, Evangelia A. Karagianni ${ }^{2, *}$ (D), Michael E. Fafalios ${ }^{2}$ and \\ Ioannis A. Koukos ${ }^{2}$ \\ 1 National and Kapodistrian University of Athens, Department of Informatics and Telecommunication \\ Panepistimiopolis, Ilisia, 15784 Athens, Greece; georgekasa@gmail.com \\ 2 Hellenic Naval Academy, Hatzikyriakou Ave., 18539 Piraeus, Greece; fafalios@hna.gr (M.E.F.); \\ koukos@hna.gr (I.A.K.) \\ * Correspondence: evka@hna.gr; Tel.: +30-210-458-1606
}

Received: 14 December 2017; Accepted: 8 February 2018; Published: 14 February 2018

\begin{abstract}
The recently modified Pascal function is further exploited in this paper in the design of passive analog filters. The Pascal approximation has non-equiripple magnitude, in contrast of the most well-known approximations, such as the Chebyshev approximation. A novelty of this work is the introduction of a precise method that calculates the coefficients of the Pascal function. Two examples are presented for the passive design to illustrate the advantages and the disadvantages of the Pascal approximation. Moreover, the values of the passive elements can be taken from tables, which are created to define the normalized values of these elements for the Pascal approximation, as Zverev had done for the Chebyshev, Elliptic, and other approximations. Although Pascal approximation can be implemented to both passive and active filter designs, a passive filter design is addressed in this paper, and the benefits and shortcomings of Pascal approximation are presented and discussed.
\end{abstract}

Keywords: analog filters; pascal approximation; pascal coefficients; pascal filters; passive filters; filter approximation

\section{Introduction}

The Pascal approximation was first introduced in [1]. However, this approximation lacked one basic attribute all polynomial approximations used in filter design should share, namely the value of 1 at $\Omega=1$. Furthermore, a new modified Pascal function with the above feature was introduced in [2], where a filter-appropriate modified symmetric Pascal function was acquired after some manipulation of the Pascal function. This later form of Pascal function is exploited further here.

The Pascal approximation [1-4], like other similar approximations used in filter design, uses a polynomial as the approximating function in the filter gain response

$$
\mathrm{G}(\Omega)=\frac{\mathrm{H}_{0}}{\sqrt{1+\lambda^{2} \mathrm{P}_{\mathrm{D}}(\mathrm{N}, \Omega)^{2}}}
$$

where $\mathrm{N}$ is the order of the filter, $\lambda$ is the ripple factor, corresponding to the $\varepsilon$ ripple factor of Chebyshev approximation, $\mathrm{H}_{0}$ is the maximum permitted passband gain and $\mathrm{P}_{\mathrm{D}}(\mathrm{N}, \Omega)$ is the modified Pascal polynomials of degree $\mathrm{N}$ from $[3,4]$

$$
\mathrm{P}_{\mathrm{D}}(\mathrm{N}, \Omega)=\frac{(-1)^{\mathrm{N}}}{\mathrm{N} !} \prod_{\mathrm{k}=1}^{\mathrm{N}}\left(\frac{\mathrm{N}+1}{2} \Omega \Omega_{\mathrm{D}}+\frac{\mathrm{N}-1}{2}-\mathrm{k}+1\right)
$$


The frequency $\Omega_{\mathrm{D}}$ is the frequency where the shifted and scaled symmetric Pascal function takes the value $-P_{D \max }$, where $P_{\text {Dmax }}$ is the higher local maximum in the interval $(-1,1)$. Both variables are independent of $\Omega$ and they depend solely on the order of the filter $(\mathrm{N})$. Their values for range of filter orders $\mathrm{N}$ is tabulated in [3].

The ripple factor $\lambda$ is bounded between $\lambda_{\min }$ and $\lambda_{\max }$ as presented in Figure 1 . The two bounds can be expressed either in linear form, as functions of the gains $\left(\mathrm{H}_{0}, \mathrm{H}_{\mathrm{c}}, \mathrm{H}_{\mathrm{s}}\right)$ or in the logarithmic form of logarithmic, as functions of the attenuation bounds $\mathrm{A}_{\max }$ and $\mathrm{A}_{\min }$ both bounds are positive

$$
\frac{\sqrt{10^{\frac{A_{\min }}{10}}-1}}{\left|\mathrm{P}_{\mathrm{D}}\left(\mathrm{N}, \Omega_{\mathrm{s}}\right)\right|}=\frac{\sqrt{\left(\frac{\mathrm{H}_{0}}{\mathrm{H}_{\mathrm{s}}}\right)^{2}-1}}{\left|\mathrm{P}_{\mathrm{D}}\left(\mathrm{N}, \Omega_{\mathrm{s}}\right)\right|}=\lambda_{\min } \leq \lambda \leq \lambda_{\max }=\frac{\sqrt{\left(\frac{\mathrm{H}_{0}}{\mathrm{H}_{\mathrm{c}}}\right)^{2}-1}}{\left|\mathrm{P}_{\mathrm{D}}(\mathrm{N}, 1)\right|}=\frac{\sqrt{10^{\frac{\mathrm{A}_{\max }}{10}}-1}}{\left|\mathrm{P}_{\mathrm{D}}(\mathrm{N}, 1)\right|}, \mathrm{P}_{\mathrm{D}}(\mathrm{N}, 1)=\mathrm{P}_{\operatorname{Dmax}}
$$

For the calculation of the filter order $\mathrm{N}$, as (4) cannot be solved analytically for $\mathrm{N}$, can be determined either from the nomograph in [3] or from the inequality

$$
\left|\frac{\mathrm{P}_{\text {Dmax }}}{\mathrm{P}_{\mathrm{D}}\left(\mathrm{N}, \Omega_{\mathrm{S}}\right)}\right| \leq \mathrm{g}=\sqrt{\frac{\left(\frac{\mathrm{H}_{0}}{\mathrm{H}_{\mathrm{c}}}\right)^{2}-1}{\left(\frac{\mathrm{H}_{0}}{\mathrm{H}_{\mathrm{s}}}\right)^{2}-1}}=\sqrt{\frac{10^{\frac{A_{\max }}{10}}-1}{10^{\frac{A_{\min }}{10}}-1}}
$$
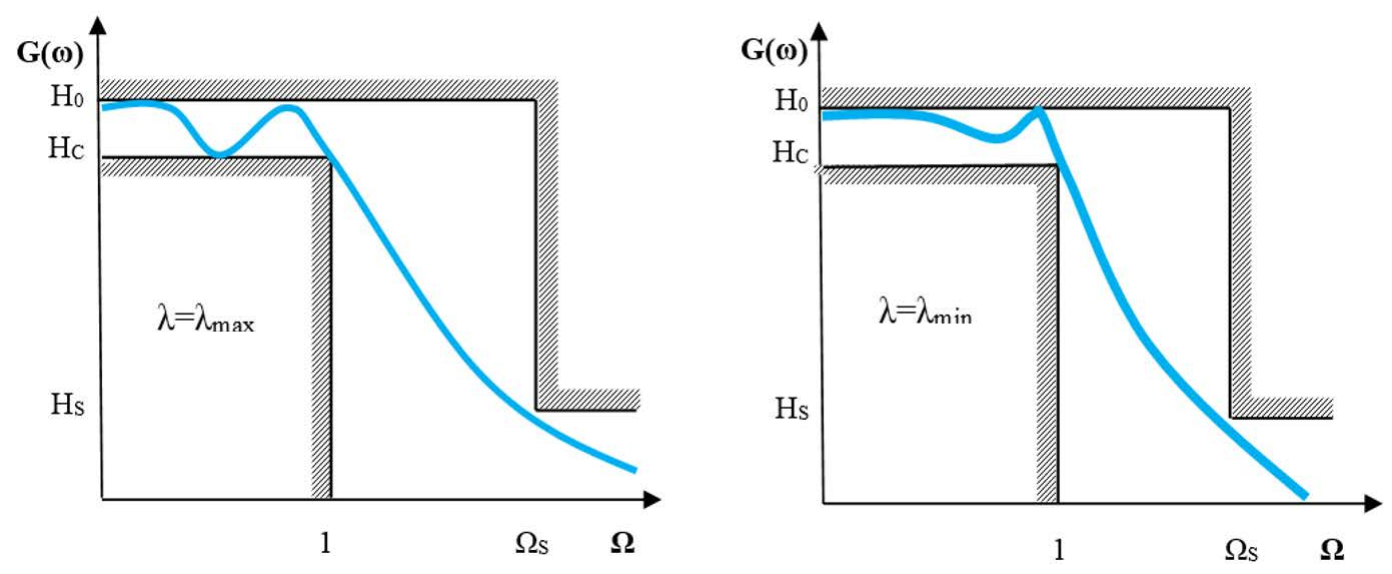

Figure 1. Choices of ripple factor $\lambda$, for $\mathrm{N}_{\text {Pascal }}=7$.

In the above inequality, the only unknown is $\mathrm{N}$ therefore, order $\mathrm{N}$ can be calculated using a numerical software tool (e.g., Mathcad, MATLAB, etc.). As it has been shown in [3], under the best case scenario, the order of the Pascal polynomial is the same with the order of the Chebyshev one (especially for low order filters) while under the worst case scenario it will be $\mathrm{N}_{\text {Cheb }}+2$.

The transfer function of the filter can be derived from the squared magnitude response $|\mathrm{H}(\mathrm{j} \omega)|^{2}$

$$
\mathrm{H}(\mathrm{s}) \mathrm{H}(-\mathrm{s})=\frac{\mathrm{N}(\mathrm{s}) \mathrm{N}(-\mathrm{s})}{\mathrm{D}(\mathrm{s}) \mathrm{D}(-\mathrm{s})}=|\mathrm{G}(\mathrm{j} \Omega)|_{\Omega=-\mathrm{js}}^{2}=\frac{\mathrm{H}_{0}^{2}}{1+\left.\lambda^{2} \mathrm{P}_{\mathrm{D}}^{2}(\mathrm{~N}, \Omega)\right|_{\Omega=-\mathrm{js}}}
$$

Since Equation (5) is a polynomial approximation, the filter transfer function is expected to be all-pole, given that the numerator will not have any finite zeros. The poles of the transfer function cannot be calculated analytically and again a numerical software tool should be used; the poles of the filter, however, must be selected as the roots lying on the left half s-plane (Hurwitz polynomial). The transfer function of the Pascal approximation can be expressed in the form 


$$
\mathrm{H}(\mathrm{s})=\frac{\frac{\mathrm{H}_{0}}{\lambda \cdot\left|\mathrm{A}_{\mathrm{N}}\right|}}{\left(\mathrm{s}+\mathrm{s}_{\mathrm{R}}\right)^{\mathrm{h}} \prod_{\mathrm{k}=1}^{\frac{\mathrm{N}-\mathrm{h}}{2}}\left(\mathrm{~s}^{2}+2 \operatorname{Re}\left(\mathrm{s}_{\mathrm{k}}\right)+\left|\mathrm{s}_{\mathrm{k}}\right|^{2}\right)}, \mathrm{h}=\bmod (\mathrm{N}, 2)
$$

where $A_{N}$ is the leading coefficient of the polynomial $P_{D}$ and can be calculated from the Equation (3)

$$
\mathrm{A}_{\mathrm{N}}=\frac{(-1)^{\mathrm{N}}}{\mathrm{N} !}\left(\frac{\mathrm{N}+1}{2} \Omega_{\mathrm{D}}\right)^{\mathrm{N}}
$$

A more detailed version of this paper is presented in [5], where the tables that have been created, are depicting the normalized values of passive elements for $N=2-9$ for a variety of values of $A_{\max }$.

\section{Calculations of the Pascal Function Coefficients}

In contrast to the most popular approximations used in the design of electric filters, the Pascal polynomials are not orthogonal. This feature results in some difficulties in the calculation of the coefficients of the polynomial. As a result of non-orthogonality, a recursive method (Christoffel-Darboux formula) cannot be used to calculate the coefficients of the polynomials. In this paper, a novel method is proposed to calculate all the coefficients of the Pascal polynomial.

The leading coefficient can be calculated directly from Equation (7). The key step for the calculation of the rest of them is to calculate the basic terms, as in the equation

$$
\operatorname{Basic} \operatorname{Term}(\mathrm{i})=\left(\frac{2 \mathrm{i}+1+\mathrm{h}}{2}\right)^{2}, \mathrm{i}=0,1, \ldots,\left(\frac{\mathrm{N}-\mathrm{h}}{2}-1\right)
$$

The coefficients can then be calculated from the unique combinations of the basic terms, while the unique combinations will be expressed as $\left(\begin{array}{c}\frac{\mathrm{N}-\mathrm{h}}{2} \\ \mathrm{k}\end{array}\right), \mathrm{k}=1,2,3, \ldots, \frac{\mathrm{N}-\mathrm{h}}{2}$ (see Appendix A for the calculation of the coefficients for $\mathrm{N}=8$ ). The equation below yields the remaining coefficients of the Pascal function

$$
\mathrm{A}_{\mathrm{N}-2 \mathrm{k}}=\frac{(-1)^{\mathrm{k}+\mathrm{h}}}{\mathrm{N} !}\left(\frac{\mathrm{N}+1}{2} \Omega_{\mathrm{D}}\right)^{\mathrm{N}-2 \mathrm{k}} \sum\left(\begin{array}{c}
\frac{\mathrm{N}-\mathrm{h}}{2} \\
\mathrm{k}
\end{array}\right)
$$

The lowest order coefficient can be calculated three alternative ways. The first alternative uses Equation (9) with $\mathrm{k}=(\mathrm{N}-\mathrm{h}) / 2$, while the second is derived from [3]

$$
A_{h}=\frac{(-1)^{\frac{N+h}{2}}}{N !}\left(\frac{N+1}{2} \Omega_{D}\right)^{h} \prod_{k=1}^{\frac{N-h}{2}}\left(\frac{N+1}{2}-k\right)^{2}
$$

The third alternative is based on Equation (10). Instead of using a product the lowest coefficient can be calculated using the Gamma function

$$
A_{h}=\frac{(-1)^{\frac{N+h}{2}}}{N !}\left(\frac{N+1}{2} \Omega_{D}\right)^{h}\left[\frac{\Gamma\left(\frac{N+1}{2}\right)}{\Gamma\left(\frac{N+1}{2}-\frac{N-h}{2}\right)}\right]^{2}
$$

The number of terms of every coefficient can be calculated from the Pascal triangle. Indeed, a Pascal triangle is formed by the either the even or the odd columns in Table 1. Alternatively, the number of terms can be obtained by the binomial coefficient as expressed below [5]

$$
\text { Number of Terms } A_{x}=\left(\begin{array}{c}
\frac{N-h}{2} \\
i
\end{array}\right), x=N-2 i, i=0,1, \ldots, \frac{N-h}{2}
$$


Table 1. Number of terms of the polynomial Pascal for every coefficient $(\mathrm{N}=2-9)$.

\begin{tabular}{ccccccccc}
\hline $\mathbf{N}$ & $\mathbf{2}$ & $\mathbf{3}$ & $\mathbf{4}$ & $\mathbf{5}$ & $\mathbf{6}$ & $\mathbf{7}$ & $\mathbf{8}$ & $\mathbf{9}$ \\
\hline $\mathrm{A}_{0}$ & 1 & & 1 & & 1 & & 1 & \\
$\mathrm{~A}_{1}$ & 1 & 1 & & 1 & & 1 & & 1 \\
$\mathrm{~A}_{2}$ & 1 & & 2 & & 3 & & 4 & \\
$\mathrm{~A}_{3}$ & & 1 & 1 & 2 & & 3 & & 4 \\
$\mathrm{~A}_{4}$ & & & & 3 & & 6 & \\
$\mathrm{~A}_{5}$ & & & & & 1 & 3 & & 6 \\
$\mathrm{~A}_{6}$ & & & & & & 1 & & 4 \\
$\mathrm{~A}_{7}$ & & & & & & & 1 & \\
$\mathrm{~A}_{8}$ & & & & & & & & 1 \\
$\mathrm{~A}_{9}$ & & & 4 & 4 & 8 & 8 & 16 & 16 \\
\hline Sum & 2 & 2 & & & & & & \\
\hline
\end{tabular}

Table 1 tabulates the number of terms of every coefficient for $\mathrm{N}=2$ to 9 ; it must be noted that the leading coefficient and the lowest order coefficient $\left(\mathrm{A}_{\mathrm{k}}\right)$ will always have only one term.

The sum of the terms of every coefficient is equal to $2^{\left(\frac{\mathrm{N}-\mathrm{h}}{2}\right)}$. It must be mentioned that the ratio of the modified Pascal polynomial $\left(\mathrm{P}_{\mathrm{D}}\right)$ over $\mathrm{P}_{\mathrm{D}}(\mathrm{N}, 1)=\mathrm{P}_{\text {Dmax }}$ for $\mathrm{N}=2$ to 3 , yields the $\mathrm{C}_{\mathrm{N}}(\Omega)$ of Chebyshev [5].

\section{Passive Filter Design through the Pascal Approximation}

The passive filter design has the form of the doubly resistively terminated two-port LC circuit, as shown in Figure 2.

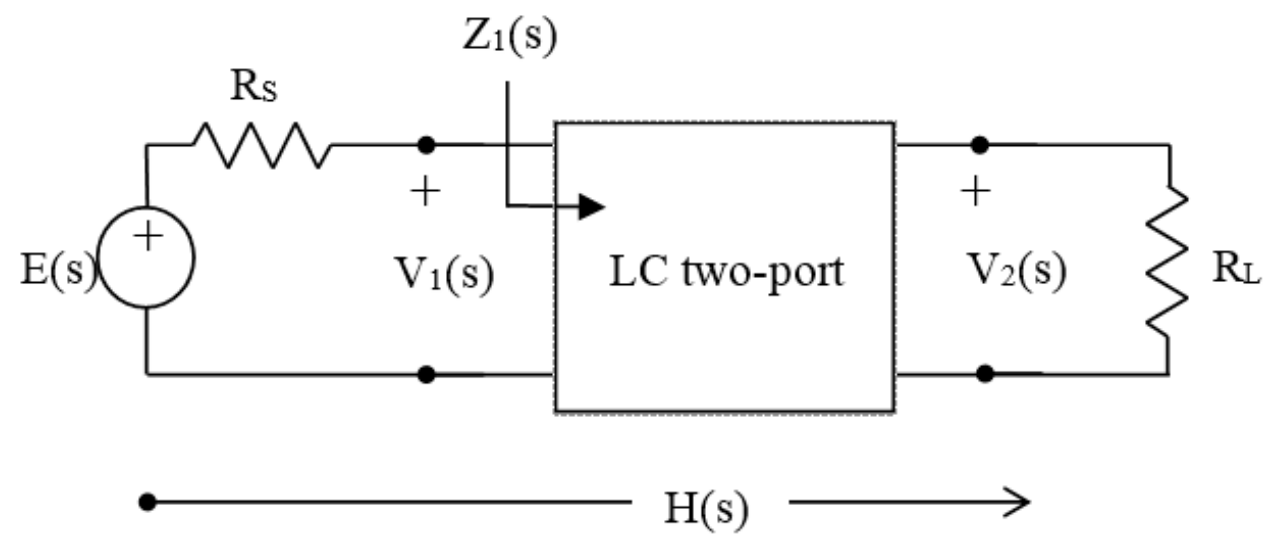

Figure 2. Resistively terminated passive two-port network at source and load [2].

The passive filters have two more specifications than the active filters, namely $R_{S}$ and $R_{L}$. Specifications are typically given in the form of effective attenuation $\mathrm{A}(\Omega)$

$$
\mathrm{A}(\Omega)=10 \log \left(\frac{\mathrm{P}_{\max }}{\mathrm{P}_{2}(\Omega)}\right)=20 \log \left(\frac{1}{2} \sqrt{\frac{\mathrm{R}_{\mathrm{L}}}{\mathrm{R}_{\mathrm{s}}}}\left|\frac{\mathrm{E}(\mathrm{j} \Omega)}{\mathrm{V}_{2}(\mathrm{j} \Omega)}\right|\right)=20 \log \left(\frac{1}{2} \sqrt{\frac{\mathrm{R}_{\mathrm{L}}}{\mathrm{R}_{\mathrm{s}}}} \frac{1}{|\mathrm{H}(\mathrm{j} \Omega)|}\right)
$$

where $P_{\max }$ is the maximum power the source can deliver and $P_{2}(\Omega)$ is the average power of the load $\left(R_{L}\right)$. Effective attenuation $\mathrm{A}(\Omega)$ is assumed to have only positive values; it becomes zero at the frequencies of maximum power transfer.

The attenuation at $\mathrm{dc}(\Omega=0)$ is given by

$$
\mathrm{A}(0)=\mathrm{A}_{0}=20 \log \left(\frac{\mathrm{R}_{\mathrm{S}}+\mathrm{R}_{\mathrm{L}}}{2 \sqrt{\mathrm{R}_{\mathrm{L}} \mathrm{R}_{\mathrm{S}}}}\right)
$$


The above equation is zero only when $R_{s}=R_{L}$ and assumes only positives values for the other cases. The attenuation function $\mathrm{A}(\Omega)$ for all approximations must have $\mathrm{A}(0)=\mathrm{A}_{0}$ for the design of the passive doubly resistively terminated LC low-pass filter. The Pascal attenuation is given from [4]

$$
\mathrm{A}_{\text {Pascal }}(\Omega)=\mathrm{A}_{0}-20(1-\mathrm{h}) \log \left(\sqrt{1+\lambda_{\max }{ }^{2} \mathrm{P}_{\mathrm{D}}(\mathrm{N}, 0)^{2}}\right)+20 \log \left(\sqrt{1+\lambda_{\max }{ }^{2} \mathrm{P}_{\mathrm{D}}(\mathrm{N}, \Omega)^{2}}\right)
$$

Figures 3 and 4 depict the passband region of a Pascal filter for order $\mathrm{N}=6$ and 7 respectively. From Equation (15) we can make some assumptions on the minimum and maximum passband attenuation.

$$
\begin{gathered}
\text { Nodd }\left\{\begin{array}{c}
\mathrm{A}_{0}+20 \log \left(\sqrt{1+\lambda_{\max }{ }^{2} \mathrm{P}_{\mathrm{D}}(\mathrm{N}, \Omega)^{2}}\right) \\
\mathrm{A}_{0}
\end{array}\right. \\
\text { NEven }\left\{\begin{array}{c}
\mathrm{A}_{0}-\delta \mathrm{A}+20 \log \left(\sqrt{1+\lambda_{\max }{ }^{2} \mathrm{P}_{\mathrm{D}}(\mathrm{N}, \Omega)^{2}}\right), \delta \mathrm{A}=20 \log \left(\sqrt{1+\lambda_{\max }{ }^{2} \mathrm{P}_{\mathrm{D}}(\mathrm{N}, 0)^{2}}\right) \\
\mathrm{A}_{0}-\delta \mathrm{A}
\end{array}\right.
\end{gathered}
$$

The attenuation function from Equation (1) must be made equal to $A_{\text {Pascal }}(\Omega)$. If we modify the squared magnitude response

$$
|\mathrm{H}(\mathrm{j} \Omega)|^{2}=\frac{\frac{\mathrm{R}_{\mathrm{L}}}{\mathrm{R}_{\mathrm{s}}+\mathrm{R}_{\mathrm{L}}}\left(1+\lambda_{\max }{ }^{2} \mathrm{P}_{\mathrm{D}}^{2}(\mathrm{~N}, 0) \mid\right)^{1-\mathrm{h}}}{1+\lambda_{\max }{ }^{2} \mathrm{P}_{\mathrm{D}}^{2}(\mathrm{~N}, \Omega) \mid}
$$

The reflection coefficient function $\rho(\mathrm{s})$ can be determined from Feldkeller's equation, [6,7]

$$
\rho(s) \rho(-s)=1-\frac{4 R_{s}}{R_{L}} H(s) H(-s)=1-\frac{4 R_{s}}{R_{L}}|H(j \Omega)|_{\Omega^{2}=-s^{2}}^{2}
$$

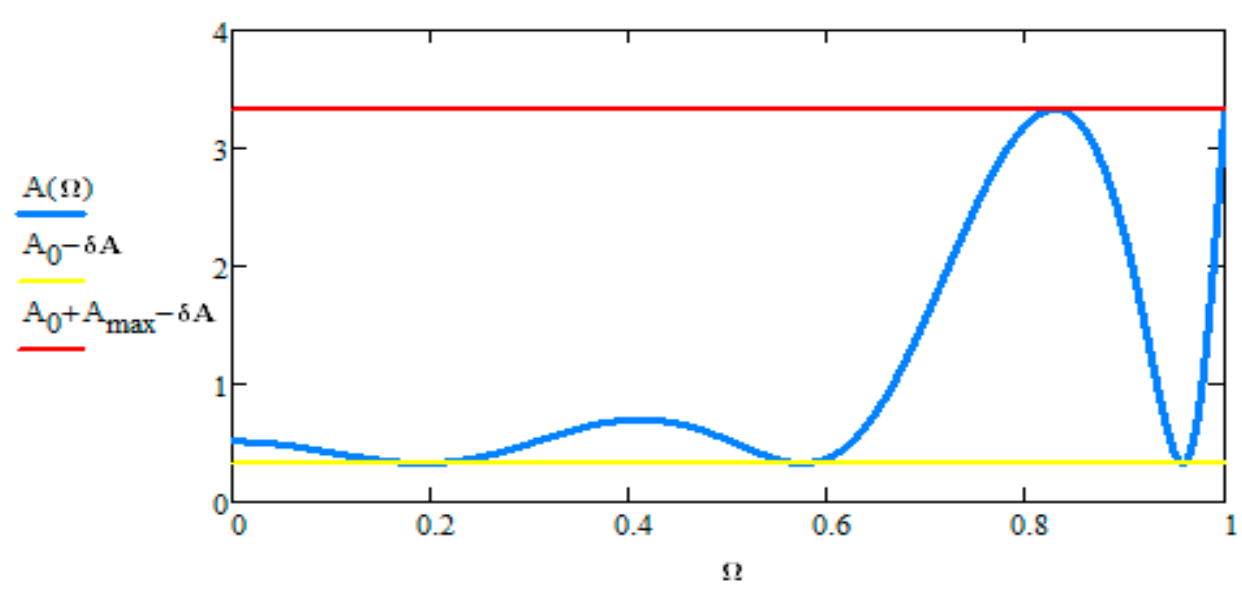

Figure 3. Passband attenuation of Low-pass Pascal filter $\mathrm{N}=6$. 


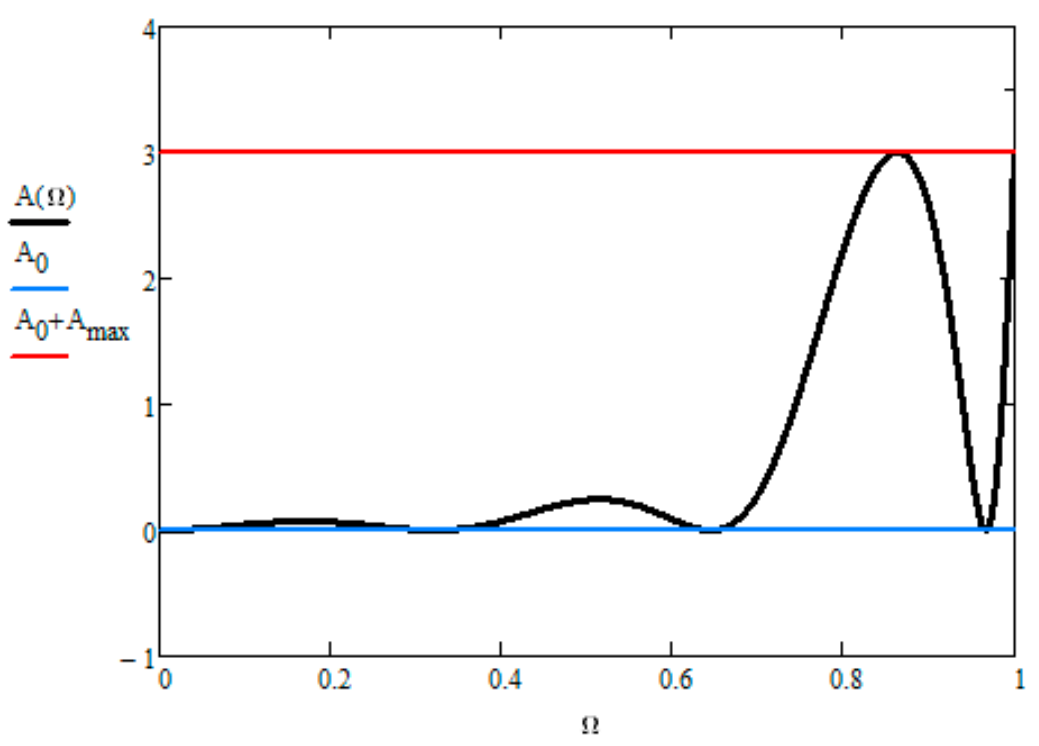

Figure 4. Passband attenuation of Low-pass Pascal filters $N=7$.

The reflection coefficient function obeys two major rules:

1. The poles of $\rho(s)$ must be a Hurwitz polynomial, i.e., the poles must lie on the left half plane of $s$ and $\rho(-s)$ must have the other poles

2. There are no restrictions on the location of the zeros to be implemented to Zin(s).

After having obtained $\rho(\mathrm{s})$ by Feldkeller equation, the input impedance, Zin(s), can be expressed as $[2,6-8]$

$$
\operatorname{Zin}(s)= \pm \frac{1-\rho(s)}{1+\rho(s)}
$$

After the calculation of Zin(s), the synthesis of the passive filter ladder can begin by removing the poles at infinity. The procedure of removing poles at infinity requires a positive real function (Zin(s)), which the degree of numerator is greater by one from the order of denominator. The passive normalized element is exported from the division between the coefficients of highest order of numerator and denominator. This process is recursively repeated for all next elements, up to the filter order $\mathrm{N}$ [6-8].

\section{Even Order Pascal Filters}

For even order filter design through the Pascal approximation, the same restriction as in the Chebyshev approximation case holds, namely, $R_{s}=R_{L}$. Indeed, if, $R_{s}=R_{L}$ then $A_{0}$ is zero and the attenuation for some frequencies is below 0 (the Attenuation by definition takes on only non-negative values). To overcome this problem, the order of the filter must be increased by one. In this paper, the term 'direct design', inherited from [4], will be used to imply that no order increase will be made and the filter can be synthesized in the usual manner.

Another problem arises when $\delta A>A_{0}$ i.e., when $R_{S 1}<R_{s}<R_{s 2}$. In that case, the filter cannot be directly designed. The design of the filter is then achieved with lower ripple at passband area. To find the range for which the filter cannot be designed directly, the roots must be calculated from the equation [4]

$$
\begin{gathered}
\mathrm{A}_{0}=20 \log \left(\frac{\mathrm{R}_{\mathrm{s}}+\mathrm{R}_{\mathrm{L}}}{2 \sqrt{\mathrm{R}_{\mathrm{s}} \mathrm{R}_{\mathrm{L}}}}\right)=20 \log \left(\sqrt{1+\lambda_{\max }{ }^{2} \mathrm{P}_{\mathrm{D}}(\mathrm{N}, 0)^{2}}\right) \\
\mathrm{R}_{\mathrm{s} 1,2}=1+2 \lambda_{\max }{ }^{2} \mathrm{P}_{\mathrm{D}}(\mathrm{N}, 0)^{2} \pm 2 \lambda_{\max } \mathrm{P}_{\mathrm{D}}(\mathrm{N}, 0) \sqrt{1+\lambda_{\max }{ }^{2} \mathrm{P}_{\mathrm{D}}(\mathrm{N}, 0)^{2}}
\end{gathered}
$$


If the $R_{\mathrm{S}}$ obtained by the specifications lies in the forbidden range, the maximum ripple $\left(\mathrm{A}_{\max 0}\right)$ that can be realized for even order passive Pascal filter, and provided that the specification $A_{\min }$ is lower than the maximum stopband specification $A_{\min 0}$, can be determined from [4]

$$
\begin{gathered}
\mathrm{A}_{0}=20 \log \left(\sqrt{1+\lambda_{\max }{ }^{2} \mathrm{P}_{\mathrm{D}}(\mathrm{N}, 0)^{2}}\right) \Leftrightarrow \mathrm{A}_{\max 0}=10 \log \left(\frac{\left(\mathrm{R}_{\mathrm{s}}-1\right)^{2} \mathrm{P}_{\text {Dmax }}{ }^{2}}{4 \mathrm{R}_{\mathrm{s}} \mathrm{P}_{\mathrm{D}}(\mathrm{N}, 0)^{2}}+1\right), \mathrm{R}_{\mathrm{L}}=1 \\
\left|\frac{\mathrm{P}_{\max }}{\mathrm{P}_{\mathrm{D}}\left(\mathrm{N}, \Omega_{\mathrm{s}}\right)}\right|=\sqrt{\frac{10^{\frac{\mathrm{A}_{\max 0}}{10}}-1}{10^{\frac{\mathrm{A}_{\min 0}}{10}}-1}} \Leftrightarrow 10^{\frac{\mathrm{A}_{\min 0}}{10}}-1=\mathrm{A}_{\min 0}=10 \log \left(\frac{\left(\mathrm{R}_{\mathrm{s}}-1\right)^{2} \mathrm{P}_{\mathrm{D}}\left(\mathrm{N}, \Omega_{\mathrm{s}}\right)^{2}}{4 \mathrm{R}_{\mathrm{s}} \mathrm{P}_{\mathrm{D}}(\mathrm{N}, 0)^{2}}+1\right)
\end{gathered}
$$

Conversely to the Chebyshev approximation case, the Pascal approximation offers the advantage that the forbidden range depends on the two constants $\left(A_{\max }, N\right)$.

In order to give a summary of the passive filter design with Pascal Approximation, the following steps are listed:

For $\mathrm{N}$ odd:

Filter design is straightforward.

For $\mathrm{N}$ even:

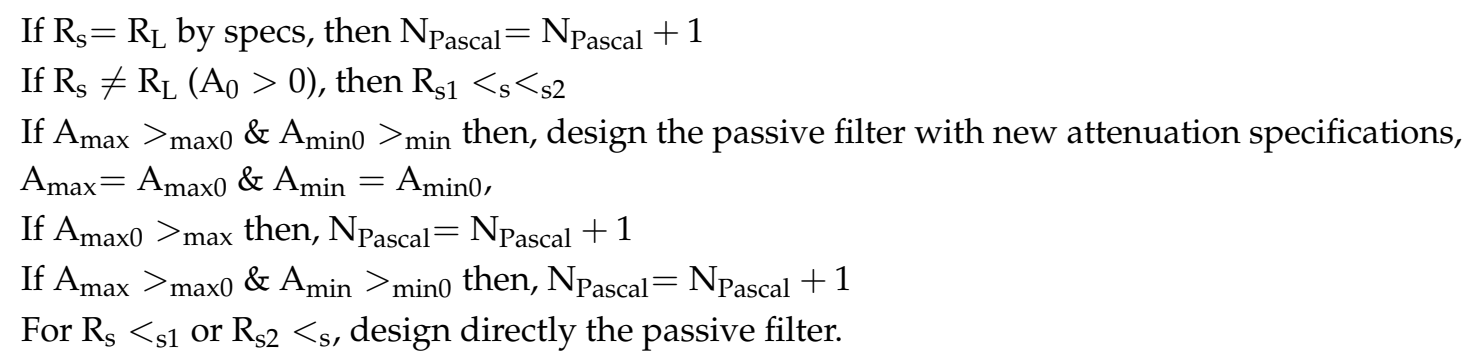

\section{Design Examples}

\subsection{Example 1}

The first example designs a low-pass filter, given the normalized specifications $\left\{\mathrm{A}_{\max }=3 \mathrm{~dB}\right.$, $\left.\mathrm{A}_{\text {min }}=55 \mathrm{~dB}, \Omega_{\mathrm{s}}=2, \mathrm{R}_{\mathrm{s}}=0.5, \mathrm{R}_{\mathrm{L}}=1\right\}$. The Pascal order $\mathrm{N}$ is calculated from Equation (4) which yields $\mathrm{N}_{\text {Pascal }}=6$, the order of the Chebyshev approximation is the same as that of the Pascal approximation and $\delta \mathrm{A}$ is $0.183\left(\mathrm{~A}_{0}>\delta \mathrm{A}\right)$. The ripple factor $\lambda_{\max }$ is 42.5 as calculated from Equation (3). In order to design the passive filter with $\lambda_{\max }$ at $\Omega_{S}$, the attenuation is greater than $A_{0}+A_{M i n}-\delta A$ and $\mathrm{A}_{0}$ is 0.512 . The forbidden range of $\mathrm{R}_{\mathrm{S}}$ for the Chebyshev approximation can be calculated from Equation (7)

$$
\mathrm{R}_{\mathrm{s} 1,2}=2 \cdot 10^{\frac{\mathrm{A}_{\max }}{10}}-1 \pm 2 \sqrt{10^{\frac{A_{\max }}{10}}\left(10^{\frac{A_{\max }}{10}}-1\right)}
$$

which yields $R_{\mathrm{s} 1}=0.1721$ and $R_{\mathrm{s} 2}=5.8089$. For the Pascal approximation as in Equation (19) also produces the order of the filter, it yields $R_{s 1 \text { Pascal }}=0.6711$ and $R_{s 2 \text { Pascal }}=1.5012$. For the specified $\mathrm{R}_{\mathrm{S}}$ the filter can be directly designed by the Pascal approximation. On the other hand, through the Chebyshev approximation, the filter has to be designed with reduced ripple in passband. From the tables given in [5] with passband ripple $A_{\max }=3 \mathrm{~dB}$, the normalized values of passive elements of the Pascal approximation can be obtained as in first and third lines of the Table 2. The next step is to de-normalize the passive elements with $\mathrm{F}_{\mathrm{c}}=500 \mathrm{MHz}, \mathrm{R}_{0}=50 \mathrm{Ohm}$ (second and fifth lines in Table 2) and then to simulate the passive filter which is presented in Figure 5 with the use of an appropriate software tool, e.g., ADS 2011. 
Table 2. Normalized and de-normalized values of passive elements for Pascal $\mathrm{N}=6$ and Amax $=3 \mathrm{~dB}$ [8].

\begin{tabular}{cccc}
\hline $\mathrm{R}_{\mathrm{s}}=0.5$ & $\mathrm{R}_{\mathrm{L}}=1$ & $\mathrm{~L}_{1}=1.6625687$ & $\mathrm{C}_{2}=2.0386647$ \\
\hline $\mathrm{R}_{\mathrm{s}}=25 \Omega$ & $\mathrm{R}_{\mathrm{L}}=50 \Omega$ & $\mathrm{L}_{1}=26.4606 \mathrm{nH}$ & $\mathrm{C}_{2}=12.9785 \mathrm{pF}$ \\
\hline $\mathrm{L}_{3}=1.6446552$ & $\mathrm{C}_{4}=1.947702$ & $\mathrm{~L}_{5}=1.8076952$ & $\mathrm{C}_{6}=1.397581$ \\
\hline $\mathrm{L}_{3}=26.1755 \mathrm{nH}$ & $\mathrm{C}_{4}=12.39946 \mathrm{pF}$ & $\mathrm{L}_{5}=28.77036 \mathrm{nH}$ & $\mathrm{C}_{6}=8.897275 \mathrm{pF}$ \\
\hline
\end{tabular}

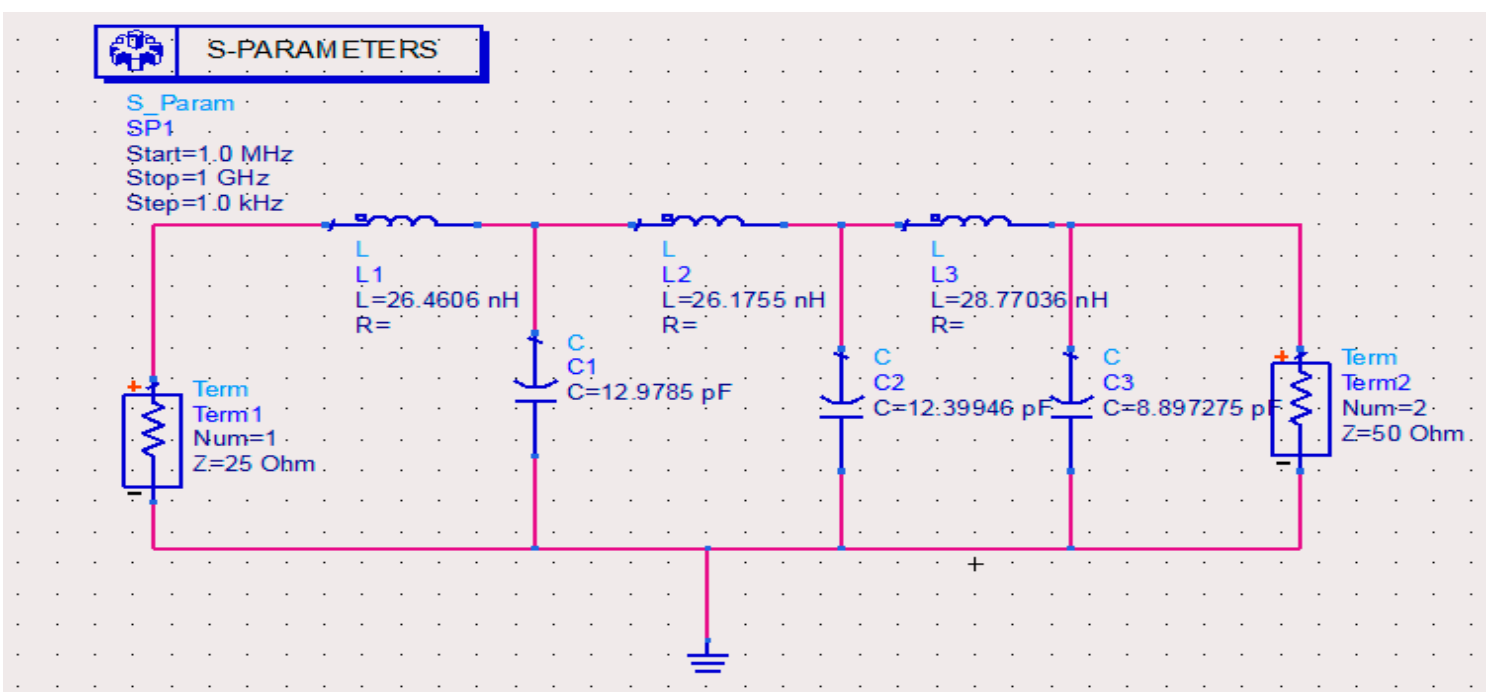

Figure 5. Low-pass filter with de-normalized passive elements simulated in ADS 2011.

Figure 6 verifies that the experimental one (red continuous line) coincides with high accuracy to the theoretical ones (grey dotted color), thus the procedure of removing the poles at infinity from Equation (8) has been successfully completed and the next step is to simulate the passive filter.

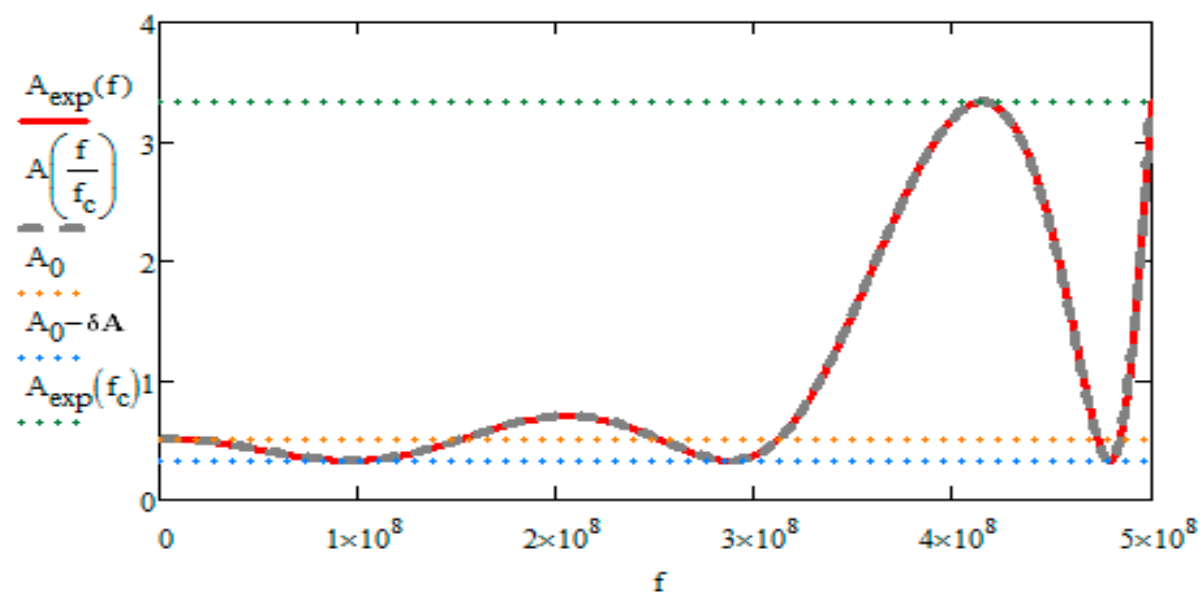

Figure 6. Passband of the Pascal Filter.

The proximity between the experimental model, obtained through simulation and the theoretical model. In theory, the attenuation at $500 \mathrm{MHz}$ must be equal to

$$
\mathrm{A}_{0}+\mathrm{A}_{\max }-\delta \mathrm{A}=20 \log \left(\frac{\mathrm{R}_{\mathrm{s}}+\mathrm{R}_{\mathrm{L}}}{2 \sqrt{\mathrm{R}_{\mathrm{S}} \mathrm{R}_{\mathrm{L}}}}\right)+\mathrm{A}_{\max }-\delta \mathrm{A}=3.328
$$


The value obtained by simulation is -3.328 (it must be highlighted that these values are of opposite signs, because ADS simulate Gain rather than Attenuation, so it holds $A(\Omega)=-G(\Omega)[2]$ ). The other parameter that should be checked is the attenuation at the edge of the stopband $\Omega_{\mathrm{s}} \times \mathrm{F}_{\mathrm{c}}$. Both the model value and the simulated value (as presented in Figure 7) for the gain are -58.787 and from the mathematical program is exactly the same. As the design has opted for maximum ripple factor $\lambda_{\max }$, the gain is expected to be less than $A_{0}+A_{\min }-\delta A=55.328$.

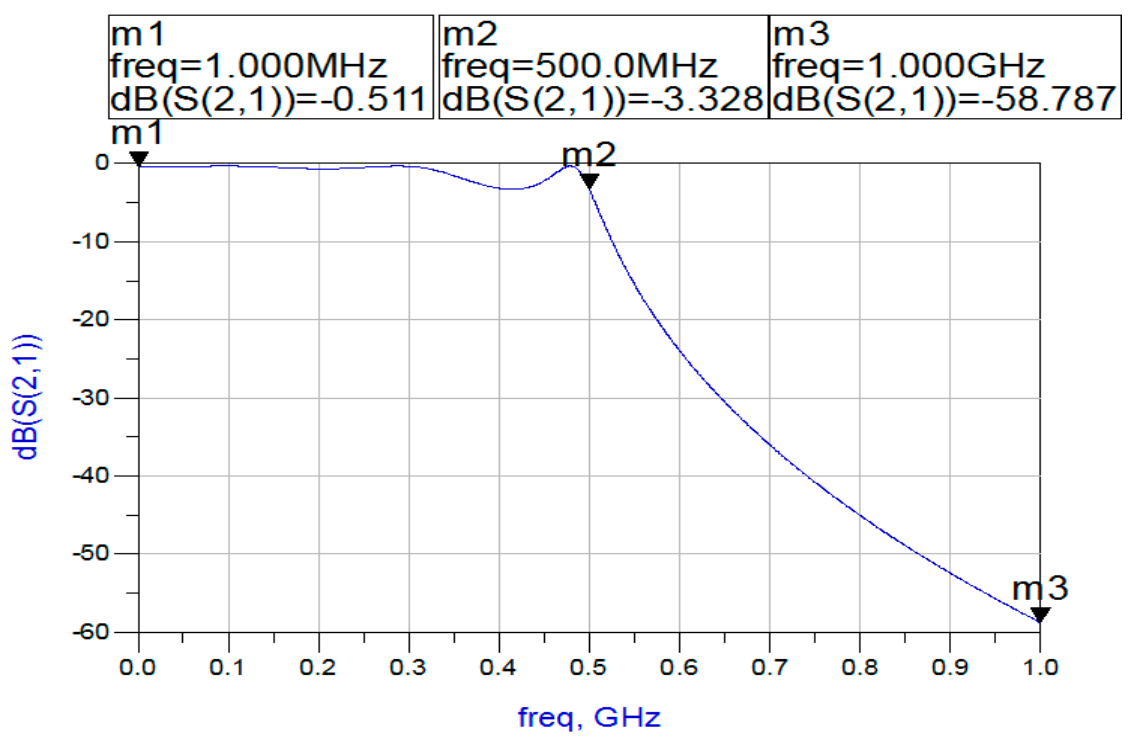

Figure 7. Simulation of low-pass filter in ADS 2011.

\subsection{Example 2}

The second design example assumes that the normalized specifications given are: $\left\{\mathrm{A}_{\max }=0.5 \mathrm{~dB}\right.$, $\left.\mathrm{A}_{\min }=55 \mathrm{~dB}, \Omega_{\mathrm{S}}=2, \mathrm{R}_{\mathrm{s}}=\mathrm{R}_{\mathrm{L}}=1\right\}$. The order $\mathrm{N}$ of Pascal approximation is calculated from Equation (4) yields $\mathrm{N}_{\text {Pascal }}=7$, while the order of the Chebyshev approximation is same. The ripple factor $\lambda_{\max }$ is 18.369 as calculated from Equation (3). For this example it has been selected to design the passive filter with $\lambda_{\max }$ at $\Omega_{s}$; the gain is therefore less than $A_{0}-A_{\min }$. The tables of [5] provide the normalized values of passive elements for the Pascal approximation, while those for the Chebyshev approximation can be obtained from [6]. Table 3 presents the normalized values of passive elements of Pascal approximation for order $\mathrm{N}=7$, in first and third lines. A minimum-inductance ladder is selected here, thus implementation will be less bulky and less expensive than constructing it with the method of minimum capacitance [7]. The next step is to de-normalize the passive elements with $\mathrm{F}_{\mathrm{C}}=500 \mathrm{MHz}, \mathrm{R}_{0}=50 \mathrm{Ohm}$ as Table 3 presents in the second and fourth lines for 7 th order Pascal filter. The last step is the filter design using the ADS 2011 software as presented in Figure 8.

Table 3. Normalized and de-normalized values of passive elements for Pascal $\mathrm{N}=7$ and Amax $=0.5 \mathrm{~dB}$ [5].

\begin{tabular}{cccc}
\hline $\mathrm{R}_{\mathrm{s}}=\mathrm{R}_{\mathrm{L}}=1$ & $\mathrm{C}_{1}=1.060450$ & $\mathrm{~L}_{2}=1.688734$ & $\mathrm{C}_{3}=1.904941$ \\
$\mathrm{R}_{\mathrm{s}}=\mathrm{R}_{\mathrm{L}}=50 \Omega$ & $\mathrm{C}_{1}=6.751037 \mathrm{pF}$ & $\mathrm{L}_{2}=26.87703 \mathrm{nH}$ & $\mathrm{C}_{3}=12.12723 \mathrm{pF}$ \\
\hline $\mathrm{L}_{4}=1.73878$ & $\mathrm{C}_{5}=1.90494$ & $\mathrm{~L}_{6}=1.688734$ & $\mathrm{C}_{7}=1.060450$ \\
$\mathrm{~L}_{4}=27.6736 \mathrm{nH}$ & $\mathrm{C}_{5}=12.12723 \mathrm{pF}$ & $\mathrm{L}_{6}=26.87703 \mathrm{nH}$ & $\mathrm{C}_{7}=6.751037 \mathrm{pF}$ \\
\hline
\end{tabular}




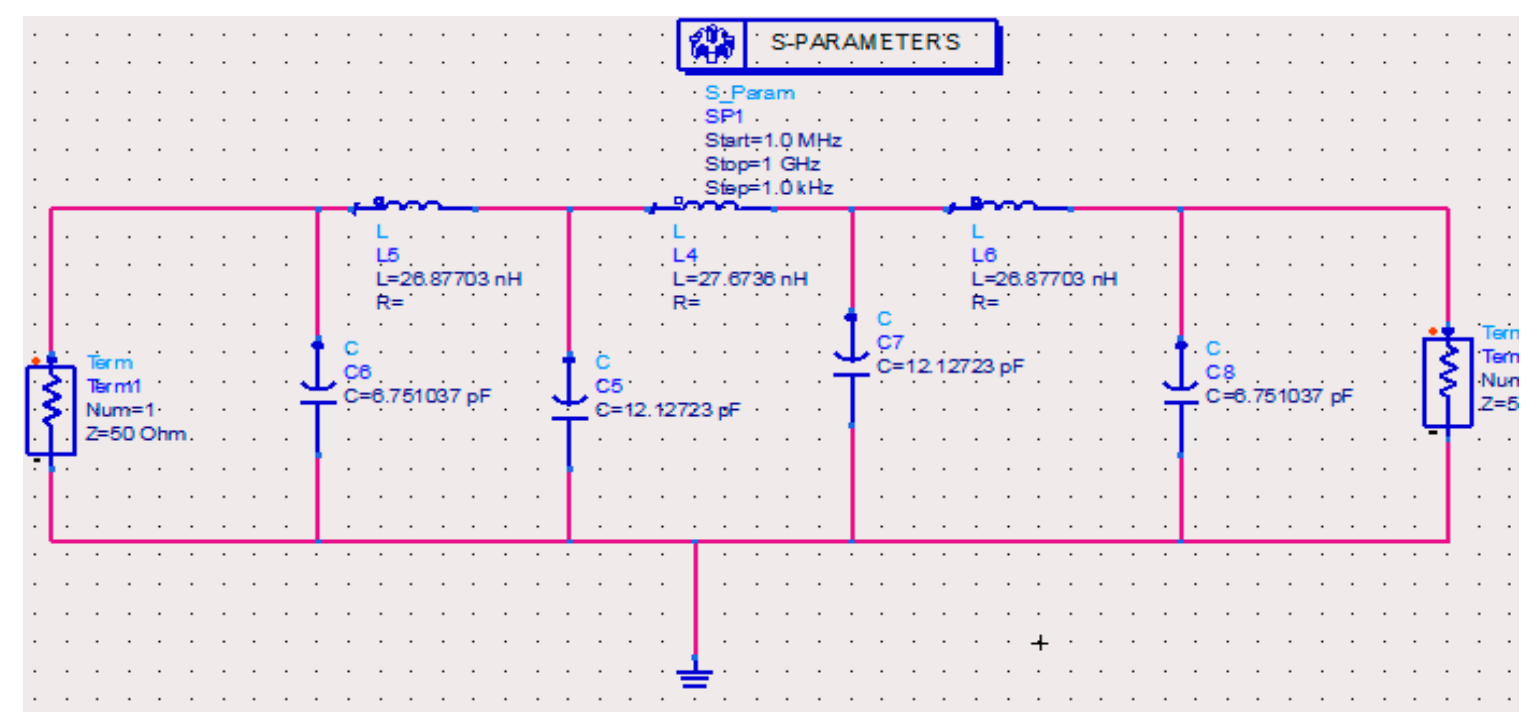

Figure 8. Low-pass Pascal filter with de- normalized passive elements, in ADS 2011.

Figure 9 verifies that the theoretical model (blue dotted line) coincides with the experimental (red continuous line). The grey dotted line shows the passband attenuation of Chebyshev approximation. The normalized values of the passive elements are founded by removing the poles at infinity from Equation (18).

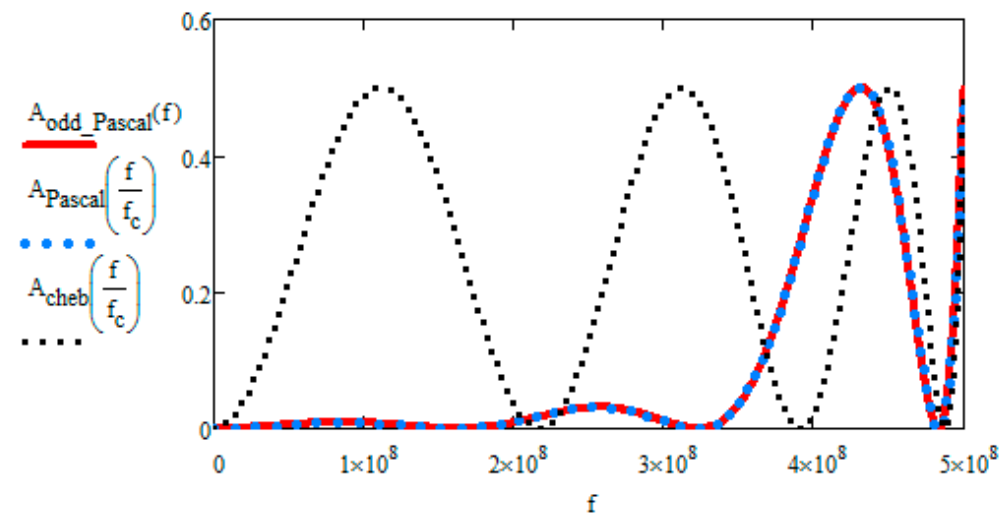

Figure 9. Passband of the Pascal and Chebyshev filters.

Once again, the experimental results coincide with the theoretical ones with high accuracy. In theory, the attenuation at $500 \mathrm{MHz}$ must be equal to

$$
\mathrm{A}_{0}+\mathrm{A}_{\max }=20 \log \left(\frac{\mathrm{R}_{\mathrm{s}}+\mathrm{R}_{\mathrm{L}}}{2 \sqrt{\mathrm{R}_{\mathrm{S}} \mathrm{R}_{\mathrm{L}}}}\right)+\mathrm{A}_{\max }=0.5
$$

The value obtained by simulation is -0.5 as presented in Figure $10\left(\mathrm{~m}^{2}\right.$ point), which is the precise theoretically obtained value. The other parameter to be checked is the attenuation at the edge of the stopband $\Omega_{\mathrm{s}}{ }^{*} \mathrm{~F}_{\mathrm{c}}$. Both the model value and the simulated value for the attenuation are -58.756 . As this design has opted for maximum ripple factor $\lambda_{\max }$, the gain is expected to be less than $A_{0}+A_{\min }=55 \mathrm{~dB}$. 


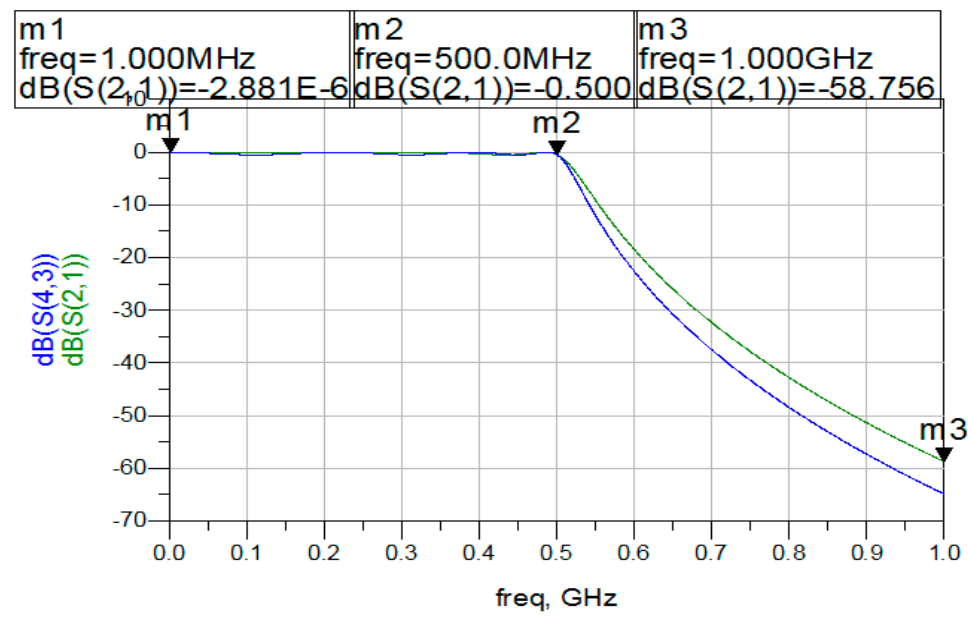

Figure 10. Simulation of low-pass filters in ADS 2011 (green: Pascal; blue: Chebyshev).

\section{Conclusions}

The Pascal all-pole approximation exhibits a non equiripple attenuation in the passband. On the other hand, the Chebyshev approximation has equiripple magnitude passband. In this work, it has been proven and presented that the ratio of the modified Pascal polynomial $P_{D}$ over $P_{D m a x}$ yields the $\mathrm{C}_{\mathrm{n}}$ of Chebyshev, for orders $\mathrm{N}=2$ to 3 .

The Pascal approximation has two drawbacks, the calculation of the order $(\mathrm{N})$ has to be done with the assistance of a numerical program and the location of the poles cannot be derived analytically. The filter order and the poles of for a $\mathrm{N}>3$ had to be calculated by numerical methods (software).

A novel method to calculate all the coefficients of the Pascal approximation polynomial has been proposed and tested through simulation in this paper, the results between the theoretical and the simulated values not only converge on given specifications, but they are the results are identical, with a high accuracy (of nine decimals, as tested). So, an analytical pattern has been established to derive the coefficients of the Pascal function. For all other cases, a numerical software tool is necessary in order to find the roots of the filter, which must lie on the left half of the complex plane.

The passive design in general, has similar difficulties in the design of odd or even-order passive filters. The superiority of the Pascal approximation, however, is that the forbidden range for $R_{S}$ is much narrower than the Chebyshev approximation for even-order passive filters design. This is owing to the fact that Equation (20) depends not only from the passband ripple, but from the order of the filter as well. In contrary, on the Chebyshev design the forbidden range depends solely from the passband ripple; which results in the forbidden range being wider than the Pascal.

Indirectly, through the examples presented in this paper, the values of the normalized elements have been acquired from created tables for a given order and a given $A_{\text {max }}$. The same tables have been created by Zverev but not for the Pascal approximation. The roll-off of the Chebyshev is steeper than the Pascal approximation.

Author Contributions: M.E.F. and E.A.K. both contributed in organizing the research. I.A.K. contributed in setting up the theoretical background and supporting this research. E.A.K. contributed in setting up the experimental background and comparing the simulation results with the ADS environment with the theoretical models. G.B.K. contributed in setting up the theoretical basis with the Pascal function and running simulations in MATLAB and ADS environment. G.B.K. and E.A.K. both contributed to the writing of this paper.

Conflicts of Interest: The authors declare no conflict of interest.

\section{Appendix A}

The leading coefficient can be calculated directly from (7), because will calculate the coefficients of $N=8$ and from [3] we can get the constant $\Omega_{D}$ for $N=8 \Omega_{D}$ is 0.79978194 and the value of $h$ is 
zero. The basic terms will be determined from $(8) \mathrm{i}=0,1, \ldots,\left(\frac{\mathrm{N}-\mathrm{h}}{2}-1\right)=0,1,2,3$ Basic Term $(\mathrm{i})=$ $\left(\frac{2 \mathrm{i}+1+\mathrm{h}}{2}\right)^{2}=\left(\frac{1}{2}\right)^{2},\left(\frac{3}{2}\right)^{2},\left(\frac{5}{2}\right)^{2},\left(\frac{7}{2}\right)^{2}$.

From (9), we will calculate the $\mathrm{A}_{\mathrm{N}-2}=\mathrm{A}_{6}$ coefficient and $\mathrm{k}=1$.

The other coefficients will be calculated with the unique combinations of basic terms of calculations of $\mathrm{A}_{4} \mathrm{k}=2$.

First will compute the unique combinations of basic terms for $k=2$, the number of terms of the specific coefficient can be calculated from the binomial coefficients yields

$$
\begin{gathered}
\left(\begin{array}{c}
\frac{\mathrm{N}-\mathrm{h}}{2} \\
\mathrm{k}
\end{array}\right)=\left(\begin{array}{c}
4 \\
2
\end{array}\right)=6 \\
\sum\left(\begin{array}{c}
\frac{\mathrm{N}-\mathrm{h}}{2} \\
\mathrm{k}
\end{array}\right)=\left(\frac{1}{2}\right)^{2}\left(\frac{3}{2}\right)^{2}+\left(\frac{1}{2}\right)^{2}\left(\frac{5}{2}\right)^{2}+\left(\frac{1}{2}\right)^{2}\left(\frac{7}{2}\right)^{2}+\left(\frac{3}{2}\right)^{2}\left(\frac{5}{2}\right)^{2}+\left(\frac{3}{2}\right)^{2}\left(\frac{7}{2}\right)^{2}+\left(\frac{5}{2}\right)^{2}\left(\frac{7}{2}\right)^{2}=123.375
\end{gathered}
$$

Easily now we can calculate $\mathrm{A}_{4}$ from the Equation (10), yielding

$$
\mathrm{A}_{4}=\frac{(-1)^{2}}{\mathrm{~N} !}\left(\frac{\mathrm{N}+1}{2} \Omega_{\mathrm{D}}\right)^{4}\left(\begin{array}{c}
\frac{\mathrm{N}-\mathrm{h}}{2} \\
\mathrm{k}
\end{array}\right)=0.5133849
$$

The same procedure will be used for the $\mathrm{A}_{2} \mathrm{k}=3:\left(\begin{array}{c}\frac{\mathrm{N}-\mathrm{h}}{2} \\ \mathrm{k}\end{array}\right)=\left(\begin{array}{l}4 \\ 3\end{array}\right)=4$

$$
\begin{gathered}
\sum\left(\begin{array}{c}
\frac{\mathrm{N}-\mathrm{h}}{2} \\
\mathrm{k}
\end{array}\right)=\left(\frac{1}{2}\right)^{2}\left(\frac{3}{2}\right)^{2}\left(\frac{5}{2}\right)^{2}+\left(\frac{1}{2}\right)^{2}\left(\frac{3}{2}\right)^{2}\left(\frac{7}{2}\right)^{2}+\left(\frac{1}{2}\right)^{2}\left(\frac{5}{2}\right)^{2}\left(\frac{7}{2}\right)^{2}=201.813 \\
\mathrm{~A}_{2}=\frac{(-1)^{3}}{\mathrm{~N} !}\left(\frac{\mathrm{N}+1}{2} \Omega_{\mathrm{D}}\right)^{2}\left(\begin{array}{c}
\frac{\mathrm{N}-\mathrm{h}}{2} \\
\mathrm{k}
\end{array}\right)=-0.0648329
\end{gathered}
$$

for the last coefficient, we can calculate three equal ways

1. $\mathrm{K}=4,\left(\begin{array}{c}\frac{\mathrm{N}-\mathrm{h}}{2} \\ \mathrm{k}\end{array}\right)=\left(\begin{array}{l}4 \\ 4\end{array}\right)=1, \sum\left(\begin{array}{c}\frac{\mathrm{N}-\mathrm{h}}{2} \\ \mathrm{k}\end{array}\right)=\left(\frac{1}{2}\right)^{2}\left(\frac{3}{2}\right)^{2}\left(\frac{5}{2}\right)^{2}\left(\frac{7}{2}\right)^{2}=43.066$,

2. $\mathrm{A}_{0}=\frac{(-1)^{4}}{\mathrm{~N} !}\left(\frac{\mathrm{N}+1}{2} \Omega_{\mathrm{D}}\right)^{0}\left(\begin{array}{c}\frac{\mathrm{N}-\mathrm{h}}{2} \\ \mathrm{k}\end{array}\right)=1.0681152 \times 10^{-3}$

3. Using the Equation (10) $A_{0}=\frac{(-1)^{\frac{N}{2} \mathrm{~h}}}{\mathrm{~N} !}\left(\frac{\mathrm{N}+1}{2} \Omega_{\mathrm{D}}\right)^{\mathrm{h}} \prod_{\mathrm{k}=1}^{\frac{\mathrm{N}-\mathrm{h}}{2}}\left(\frac{\mathrm{N}+1}{2}-\mathrm{k}\right)^{2}=1.0681152 \times 10^{-3}$

4. Or using the gamma function

5. $\mathrm{A}_{0}=\frac{(-1)^{\frac{\mathrm{N}+\mathrm{h}}{2}}}{\mathrm{~N} !}\left(\frac{\mathrm{N}+1}{2} \Omega_{\mathrm{D}}\right)^{\mathrm{h}}\left[\frac{\Gamma\left(\frac{\mathrm{N}+1}{2}\right)}{\Gamma\left(\frac{\mathrm{N}+1}{2}-\frac{\mathrm{N}-\mathrm{h}}{2}\right)}\right]^{2}=1.0681152 \times 10^{-3}$

\section{References}

1. Goodman, T.J.; Aburdene, M.F. Pascal Filters. IEEE Trans. Circuits Syst. I Regul. Pap. 2008, 55, 3090-3094. [CrossRef]

2. Dimopoulos, H.G. Analog Electronic Filters Theory, Design and Synthesis; Springer: London, UK, 2012; pp. 77-99.

3. Dimopoulos, H.G. Even-Order passive filters: Pascal versus Chebyshev. Int. J. Circuit Theory Appl. 2013, 41, 946-959. [CrossRef]

4. Dimopoulos, H.G.; Sarri, E. The modified Pascal polynomial approximation and filter design method. Int. J. Circuit Theory Appl. 2012, 40, 145-163. [CrossRef] 
5. Kasapoglou, G. Design and Implementation of a Bandpass Filter for Microwave Frequencies by the Pascal Approximation. Master's Thesis, National and Kapodistrian University of Athens, Athens, Greece, 2017. Available online: https:/ / pergamos.lib.uoa.gr/uoa/dl/frontend/browse.html?p.id=1333175 (accessed on 13 December 2017). (In Greek)

6. Sedra, A.S.; Bracket, P.O. Filter Theory and Design: Active and Passive; Pitman: London, UK, 1978; pp. 116-168.

7. Blinchkoff, H.J.; Zverev, A.I. Filtering in the Time and Frequency Domains; Noble Publishing Associates: Vancouver, WA, USA, 1986; pp. 321-322.

8. Schaumann, R.; Van Valkenburg, M.E. Design of Analog Filters; Oxford University Press: New York, NY, USA, 2001; pp. 482-524.

(C) 2018 by the authors. Licensee MDPI, Basel, Switzerland. This article is an open access article distributed under the terms and conditions of the Creative Commons Attribution (CC BY) license (http:/ / creativecommons.org/licenses/by/4.0/). 